\title{
Civilisations
}

Revue internationale d'anthropologie et de sciences

humaines

59-1 | 2010

Sexualités : apprentissage et performance

\section{La socialisation du désir homosexuel masculin à Bamako}

Christophe Broqua

\section{OpenEdition \\ Journals}

Édition électronique

URL : http://journals.openedition.org/civilisations/2242

DOI : 10.4000/civilisations. 2242

ISSN : 2032-0442

\section{Éditeur}

Institut de sociologie de l'Université Libre de Bruxelles

\section{Édition imprimée}

Date de publication : 28 juin 2010

Pagination : $37-58$

ISBN : 2-87263-029-5

ISSN : 0009-8140

Référence électronique

Christophe Broqua, "La socialisation du désir homosexuel masculin à Bamako », Civilisations [En ligne], 59-1 | 2010, mis en ligne le 29 juin 2013, consulté le 01 mai 2019. URL : http:// journals.openedition.org/civilisations/2242 ; DOI : 10.4000/civilisations.2242

(c) Tous droits réservés 


\title{
La socialisation du désir homosexuel masculin à Bamako
}

\author{
Christophe BROQUA
}

\begin{abstract}
Résumé : La littérature consacrée aux comportements homosexuels dans les pays non occidentaux a parfois été critiquée pour avoir dénié la dimension libidinale de ces actes au profit de leur fonction symbolique ou instrumentale. C'est tout particulièrement le cas des écrits qui ont été consacrés aux pratiques homosexuelles en Afrique, rarement envisagées sous l'angle du désir sexuel ou des sentiments. À partir des résultats d'une enquête ethnographique réalisée entre 2003 et 2008 auprès d'hommes ayant des pratiques homosexuelles à Bamako, sont exposées les voies par lesquelles se forme et se donne à voir ou à entendre le désir homosexuel. Dans un contexte où la catégorie homosexuelle est généralement considérée comme ne renvoyant à aucune réalité locale mais, lorsqu'elle est connue, à un comportement occidental hautement répréhensible, se pose la question du sens qui est donné au désir homosexuel et de son impact sur la formation de l'identité sociale chez les personnes concernées. Il s'agit de rendre compte des logiques de ce désir, de ses formes d'expression possibles mais aussi des contraintes sociales qui conditionnent son accomplissement.
\end{abstract}

Mots-clés : comportement homosexuel, désir sexuel, honte, Mali, socialisation.

\begin{abstract}
The scholarly literature on homosexual practices in non-Western countries has sometimes been criticized for its deny of the libidinal dimension of such acts, which was neglected to the benefit of symbolic or instrumental functions. This has especially been the case for the literature on homosexual practices in Africa, which have only rarely been considered under the angle of desire or feelings. Drawing on ethnographic fieldwork conducted between 2003 and 2008 in Bamako among men having homosexual practices, this article shows how homosexual desire is understood and expressed in this context. Furthermore, the text explores the local meaning of homosexual desire and its impact on the identity of these men, in a society where homosexuality is largely considered as a highly reprehensible Western behaviour.
\end{abstract}

Key words: homosexual practices, sexual desire, shame, Mali, socialisation. 
L

littérature consacrée aux comportements homosexuels dans les pays non occidentaux a parfois été critiquée pour avoir dénié à ceux-ci leur dimension libidinale au profit d'une analyse de leur fonction symbolique ou instrumentale (Murray 1997). Certains auteurs ont pointé l'incapacité des anthropologues à fournir des descriptions de la composante érotique de ces activités, notamment à propos des rituels d'initiation masculine en Mélanésie (Greenberg 1988 ; Herdt et Stoller 1990). Concernant la littérature africaniste, une telle critique a été adressée par exemple à Gill Shepherd (1987) pour n'avoir montré que l'aspect vénal de la sexualité, ou aux deux principaux textes d'histoire sur les formes d'homosexualité institutionnalisée observées dans les mines d'or sud-africaine (Moodie et al. 1988 ; Harries 1990), pour s'être focalisés sur la dimension instrumentale des unions et ne pas avoir voulu accorder davantage de crédit aux éléments qui tendent à montrer l'existence du désir sexuel ou du sentiment amoureux. Il n'est d'ailleurs pas anodin de constater que les premiers travaux d'anthropologues africanistes consacrés à des pratiques que le regard occidental pouvait associer à l' " homosexualité " ont porté sur les mariages entre femmes observés dans différents pays d'Afrique, dans le cadre desquels la sexualité était réputée absente, ce qui d'ailleurs a été plus tard mis en doute (Carrier et Murray 1998). Plus largement, dans de nombreux travaux ethnographiques, Jeffery P. Dennis montre que le désir « homo-érotique » est évacué au moyen de deux métarécits, l'un ne lui accordant aucune place, l'autre présupposant son omniprésence mais ne le discutant pas davantage (Dennis 2000).

Cette réticence à traiter de la dimension libidinale des pratiques homosexuelles renvoie au débat opposant approches essentialiste et constructionniste de l'homosexualité au sein de différentes disciplines depuis la publication du texte fondateur de Mary McIntosh (1968), qui invitait à considérer l'homosexualité non pas comme un "état », mais comme un « rôle » historiquement et culturellement situé. Selon cette approche, les comportements homosexuels dans les pays non occidentaux répondent à des significations variées qui ne correspondent généralement pas à la catégorie homosexuelle telle qu'on l'observe dans les pays d'Amérique du Nord ou d'Europe. La littérature constructionniste s'attache à mettre au jour les conditions de possibilité et les effets du processus de catégorisation. Mais depuis son apparition, elle relègue dans l'obscurité une question qui préoccupe pourtant bien des esprits, y compris chez les personnes « concernées » : celle de «l'origine » ou de la formation du désir homosexuel.

Deux raisons principales s'opposent à l'analyse de cet objet par les sciences sociales, qui s'ajoutent au fait que l'anthropologie peine plus largement à traiter des problématiques du désir et du plaisir. La première tient au tabou qui entoure la question des « causes » de l'homosexualité. Depuis l'avènement de l'approche constructionniste (qui correspond à celui de l'approche sociologique) de l'homosexualité, les recherches portent majoritairement sur les conditions sociales de l'expérience de celle-ci, mais répugnent à en retracer les conditions d'émergence et de développement individuels, la recherche des « causes » de l'homosexualité étant considérée comme relevant des disciplines psychologiques ou sociobiologiques, dont les sciences sociales œuvreraient ici précisément à se déprendre. Par souci de démarcation, le questionnement sociologique ou anthropologique respecte un principe au moins tacite selon lequel l'étiologie du désir homosexuel ne doit pas être recherchée davantage que celle du désir hétérosexuel. En somme, pour contrer l'approche psychologique, les sciences 
sociales préfèrent encore aujourd'hui rejeter la question qui l'incarne par excellence, plutôt que la reformuler, en considérant qu'il n'y a pas lieu de s'intéresser à l'émergence du désir homosexuel chez l'individu.

La seconde raison est que pour pouvoir être élucidée, la question de la formation du désir homosexuel nécessiterait idéalement que soient mobilisées de concert sciences sociales et psychologiques, ce que les chercheurs de l'une ou l'autre disciplines ne savent ou ne veulent généralement pas faire - à quelques exceptions près (Stein 1989 ; Epstein 1991) -, à plus forte raison dans le cadre d'une perspective transculturelle. En outre, cela pourrait inciter à renouer avec certaines approches culturalistes, qui ont précisément œuvré à combiner outils anthropologiques et psychologiques, en cherchant à éclairer des domaines de la vie sociale largement délaissés par la suite, dont bien sûr celui de la sexualité ; or de telles approches sont aujourd'hui frappées de disgrâce. Pourtant, l'un des intérêts que peut présenter l'analyse anthropologique du développement sexuel, pour peu qu'elle sache tenir compte des apports de la psychologie et de l'anthropologie culturaliste tout en les dépassant, est de permettre la prise en considération des contraintes spécifiques à l'environnement social et culturel de la sexualité (Herdt 2004).

Enfin, une difficulté supplémentaire se greffe aux précédentes, qui concerne la définition et le sens de l'objet. Loin d'être neutre, la notion de désir porte l'empreinte des sciences médicales et psychologiques qui en ont fait un des instruments de la définition et du contrôle des sujets, ce que Michel Foucault résume en une formule : «Dis-moi quel est ton désir, je te dirai qui tu es comme sujet. » (Foucault 1996 : 45). Pourtant, avant que Foucault n'insiste sur ses usages normatifs, la notion de " désir homosexuel » avait été investie d'une signification voulue transgressive par les analyses de Guy Hocquenghem, qui entendait précisément l'employer comme une arme contre l'ordre sexuel et social dominant (Hocquenghem 1972). Dans cet article, le désir homosexuel sera envisagé dans un sens plus comportemental que dispositionnel : il désignera le sentiment (au moins en partie érotique) associé à un engagement volontaire dans une pratique homosexuelle répétée.

Contrairement à ce que pourrait laisser croire le fait que les sciences sociales aient délaissé l'analyse de la formation du désir homosexuel, sans affronter les difficultés qu'elle suppose, cette question fait incontestablement sens pour ces disciplines dès lors qu'est évitée une conception essentialiste de la sexualité. Sans en faire l'objet exclusif de ce texte, je l'utiliserai comme point de départ de mon interrogation, sur la base de l'hypothèse et de la question qui suivent. Dans certains pays occidentaux tels que la France, ceux qui éprouvent un désir sexuel pour les personnes de même sexe se trouvent progressivement associés, pour une partie d'entre eux au moins, à la catégorie homosexuelle ; cette identification structure la formation du désir comme les formes de son expression ou de son accomplissement. Au Mali, aucun processus de socialisation n'est a priori « prévu » pour éduquer au désir homosexuel ; bien au contraire, on y entend souvent que l'homosexualité est un comportement spécifique aux pays d'Europe ou d'Amérique du Nord. Dès lors, comment un tel désir peut-il naître, puis se développer, dans un contexte où la catégorie homosexuelle est réputée absente (Epprecht 2008) ? J'examinerai donc ici ce qu'il en est des voies du désir homosexuel masculin à Bamako. Pour appréhender cet objet, j'ai fait le choix d'une optique comparative, visant à mettre prioritairement en perspective les situations maliennes et françaises, en raison à la fois des différences ordinairement reconnues, mais aussi du lien historique qui unit ces 
deux pays, faisant de la France, dans bien des domaines, un modèle de référence (positive ou négative) pour les Maliens.

Ce texte repose sur les résultats d'une enquête effectuée à Bamako au moyen d'observations ethnographiques, lors de séjours d'une durée totale de trois années, entre 2003 et 20081. Elle a débuté suite à la découverte de " maquis » (bars) fréquentés entre autres par des hommes ayant des pratiques homosexuelles, où de premiers contacts ont été noués. J'ai ensuite intégré divers réseaux d'interconnaissance et côtoyé plus particulièrement certains individus. Parallèlement, des relations ont été établies par le biais de sites de rencontre sur internet. Enfin, à cela s'est ajouté ma participation à une étude coordonnée par l'association Arcad-sida (Sylla et al. 2007), dans le cadre de laquelle ont été recueillis les entretiens cités ici, qui ont été réalisés en langue bambara (la langue véhiculaire au Mali) puis transcrits en Français par les enquêteurs. Les hommes rencontrés dans ces deux contextes résident tous à Bamako et appartiennent généralement à des milieux très modestes.

\section{L' entrée dans la sexualité entre hommes}

Deux caractéristiques importantes des sexualités entres hommes à Bamako doivent être soulignées d'emblée. Tout d'abord, parmi les hommes qui ont des pratiques homosexuelles, ceux qui n'ont jamais eu de relation sexuelle avec une femme sont très rares, sous doute en raison de la forte pression sociale qui s'exerce dans ce sens, en particulier de la part des pairs. Selon les résultats d'une enquête quantitative réalisée au Mali en 1998, près de la moitié des jeunes hommes vivant en milieu urbain donnent comme raison de leur premier rapport sexuel la pression sociale, exercée non seulement par les pairs masculins, mais aussi par les filles qui, selon certains des garçons interrogés, inciteraient leurs partenaires aux rapports sexuels dans le but de bénéficier des rétributions financières ou matérielles qu'ils impliquent (Gueye et al. 2001). Chez les hommes ayant des pratiques homosexuelles qui ont eu des partenaires féminines, on distingue ceux qui ont eu des relations sexuelles avec des femmes mais qui ont cessé au profit de pratiques homosexuelles exclusives et ceux qui ont à la fois des rapports sexuels avec des femmes et des hommes. Parmi ces derniers, qui sont les plus nombreux, beaucoup ont une partenaire féminine attitrée. Généralement, ceux qui n'ont pas (ou plus) de relations sexuelles avec des femmes sont aussi ceux qui dans le cadre des rapports sexuels entre hommes occupent le rôle " réceptif» (ou « passif »), renvoyant souvent à une position précise dans la relation et à une identité sociale déterminée. En effet - seconde caractéristique importante -, dans les discours des personnes concernées, la pratique homosexuelle suppose une répartition des individus en deux catégories définies principalement par le rôle occupé dans la relation sexuelle, mais aussi plus largement par l'identité sociale supposément associée, comme le suggère le vocabulaire des initiés : les « yoss » occupent le rôle « insertif » et sont d'apparence masculine; les « qualités » occupent le rôle « réceptif » et présentent des caractéristiques féminines. Cette dichotomie est d'ailleurs courante en Afrique ; elle a été observée dans différents pays de la région, tels que le Nigeria par exemple (Gaudio 2009). Si l'on peut aisément inférer de cette relative diversité des profils l'hypothèse qu'ils renvoient à différents modes d'entrée dans la sexualité, nulle typologie de cet ordre ne sera présentée ici, mais plutôt quelques tendances générales. 
Pour beaucoup d'hommes ayant des pratiques homosexuelles, l'entrée dans la sexualité avec une personne de même sexe s'est déroulée au moment de l'adolescent, parfois dans l'enfance. Pour d'autres, plus rares, elle a eu lieu à l'âge adulte. Il est possible de distinguer deux grandes catégories d'expériences : les uns ont débuté avec des pairs générationnels, les autres avec des personnes plus âgées. Dans la première catégorie, les premiers rapports s'effectuent avec des proches de la même classe d'âge, souvent sur un mode tout d'abord ludique. De tels comportements semblent fréquents durant l'enfance ou l'adolescence, mais seuls quelques-uns les poursuivront au delà de cette période d'initiation. Ces jeux sexuels supposent au minimum des pratiques d'attouchement ou de masturbation réciproques, avec ou sans éjaculation, ce qui dépend notamment de l'âge des partenaires, puisque ces pratiques débutent souvent avant la puberté. Ils peuvent également impliquer des actes de fellation ou de pénétration anale. Si l'on tient compte du fait qu'en milieu urbain au Mali, chez les garçons, l'âge médian au premier rapport hétérosexuel est d'environ dix-sept ans (Gueye et al. 2001), on comprend que chez beaucoup de ceux qui en ont connu, ces pratiques préadolescentes précèdent l'entrée dans la sexualité avec des filles. Toutefois, l'initiation sexuelle entre pairs générationnels peut encore s'effectuer après l'adolescence, une fois accomplie l'entrée dans la pratique hétérosexuelle.

Dans la seconde catégorie, le premier partenaire sexuel est un homme plus âgé, qui prend généralement l'initiative du rapport. Il peut avoir été rencontré au hasard des activités de la vie quotidienne. Mais il n'est pas rare qu'il s'agisse d'un proche voire un membre de la famille. Selon une enquête quantitative réalisée au Sénégal, le premier rapport homosexuel a souvent eu lieu durant l'adolescence avec un homme plus âgé, issu de l'entourage ou récemment rencontré. Pour un tiers des répondants, l'homme faisait partie de la famille élargie (Niang et al. 2003 : 504). Les auteurs citent notamment des exemples où l'initiateur est un oncle. Ce fait se rencontre également à Bamako :

C'est mon tonton qui m'a fait adhérer la pratique. [...] Il me choyait en me donnant de l'argent, souvent 1000 francs ou 500 francs [CFA]. De ce fait, je ne pouvais pas expliquer aux parents ce qu'il me demandait. Pour finir, il m'a demandé le rapport et je ne pouvais pas le refuser. Entre temps, j'ai fait la connaissance des amis homosexuels. C'est comme ça que j'ai intégré le « milieu ». (Entretien 7, 16 ans)

C'est le frère de mon père, mon tonton, qui m'a conduit dans cette pratique depuis que j'étais tout petit. Il avait environ 26 ou 28 ans. [...] Quand j'étais petit, dans la grande famille, il me déshabillait pour me faire l'amour. (Entretien 12, 22 ans)

Souvent, les premières expériences homosexuelles ont résulté d'un travail de persuasion de la part du plus âgé, où peuvent intervenir des arguments d'autorité, qui vont parfois jusqu'à la contrainte physique, voire le viol dans certains cas. Le fait d'offrir des cadeaux ou de l'argent aide à obtenir de l'individu convoité qu'il accepte la relation sexuelle (Broqua 2009a), comme dans l'exemple qui suit :

J'ai commencé à 17 ans. Il y avait quelqu'un qui était chez nous, il jouait avec moi chaque jour. Moi-même je ne connaissais pas le "milieu ». Il m'a invité un jour, on est parti chez lui, il m'a demandé de le masser. Moi je ne savais pas ce qu'il voulait en réalité, donc j'ai commencé à le masser petit à petit. C'est comme ça qu'il a commencé à me toucher, et finalement il m'a déshabillé. Quand il m'a déshabillé, je voulais refuser mais comme j'étais seul avec lui, il a commencé à me parler, à me 
dire des paroles douces et il m'a demandé si je peux l'embrasser. Moi j'ai dit que je ne connais pas ça, parce qu'à cet âge-là je ne regardais même pas les filles, je ne savais pas ce que c'est, j'étais surtout intéressé par les études, je ne connaissais pas cette chose-là, je ne regardais pas la télé ni rien, je ne connaissais même pas le fait d'embrasser quelqu'un. Donc il m'a embrassé. Après il a essayé de me faire l'amour. Ce jour-là j'ai été un peu blessé. Mais après il m'a donné de l'argent, il m'a dit de ne rien dire à personne. Il m'a donné beaucoup d'argent, si bien que j'ai creusé un trou pour le mettre dedans. C'était beaucoup. Mais j'ai eu un peu peur après. Il continuait à venir me chercher. Et à chaque fois qu'il venait, il me donnait de l'argent, et aussi des habits. Et tous les membres de la famille disaient que c'était mon ami et qu'on était bien ensemble, parce qu'il y a certaines vieilles personnes qui s'entendent bien avec les enfants. Nous sommes restés comme ça. Il avait une moto et à chaque fois il venait me chercher, et on sortait ensemble. À chaque fois qu'on sortait et qu'on partait chez lui, il essayait toujours de me faire l'amour, et finalement j'y ai pris goût, si bien que même s'il ne venait pas, moi-même je partais le chercher. C'est devenu une habitude. (Entretien 1, 22 ans)

Dans la majorité des témoignages, c'est la proposition d'un tiers qui semble avoir déclenché le passage à l'acte, comme si la personne qui témoigne n'en avait pas éprouvé le désir préalablement. En voici une autre illustration :

J'ai connu le rapport homme-femme, comme vous le voyez j'ai d'abord eu une copine fille en tant que telle depuis le lycée. Après ça, ma vie a changé. Parce que j'ai rencontré un ami qui m'a été très cher après, il m'a été vraiment cher. Je sais que vous voulez qu'on parle de ma vie sexuelle, vraiment je n'aime pas en parler trop mais s'il le faut... Je dis que j'ai connu un ami français, pas Français génétiquement mais un ami malien qui a vécu en France, qui était venu pour sa première fois au Mali. Et on se suivait, on sortait ensemble, il m'invitait très souvent. On faisait tout ensemble. Il me faisait des propositions. Et je me suis senti obligé d'accepter. Et il me faisait vraiment beaucoup de choses. J'étais contraint d'honorer ses propositions. C'est comme ça que c'est venu. (Entretien 17, 28 ans)

Finalement, ceux qui disent avoir éprouvé un tel désir avant de se trouver confrontés à une sollicitation de la part d'un tiers font figure d'exception. Parmi eux, l'auteur du témoignage qui suit est de ceux que l'on rencontre le moins souvent :

Je n'ai pas été entraîné par quelqu'un. D'ailleurs, quand j'avais 22 ans, en voyant mes amis nus, je sentais un effet sur moi. C'est à partir de là que j'ai su vraiment que mon attirance vers les hommes était naturelle. (Entretien 19, 29 ans)

\section{Désir contraint...}

Les témoignages dont quelques extraits viennent d'être livrés suggèrent fortement que le désir homosexuel se trouve enchâssé dès son émergence dans des relations de pouvoir ; le plus souvent, les premiers rapports homosexuels procèdent d'une initiative extérieure et d'une plus ou moins grande liberté d'action de la personne concernée. Le rôle de la contrainte ou de l'incitation musclée apparaît dans les entretiens avec suffisamment de récurrence pour que l'on s'y attarde.

Le processus de conversion par un tiers se retrouve dans le cas des individus initiés par des pairs de même génération, mais plus clairement encore lorsque l'initiateur est un homme plus âgé. C'est aussi dans ce second cas que l'épisode d'initiation témoigne en général d'une 
forme ou d'une autre de contrainte. Ce fait a lui aussi été décrit dans d'autres pays. Les auteurs de l'enquête sénégalaise indiquent que $43 \%$ des hommes interrogés disent avoir été violés au moins une fois « hors du foyer familial » (outside the family home) (Niang et al. 2003 : 507), laissant entendre que les viols au sein du foyer ne sont pas comptabilisés, ou ne sont pas comptabilisés comme viols, ce qui dans les deux cas suggère une fréquence tacitement reconnue du phénomène. Au Cameroun, les résultats d'une enquête plus récente montrent eux aussi la place importante de la contrainte lors du premier rapport sexuel, confirmant les observations antérieures de Charles Gueboguo (2006) : " Le rapport était souhaité pour $57,1 \%$ des répondants. $35,7 \%$ ont dit ne pas avoir souhaité le rapport sexuel mais l'avoir accepté et 7,1\% ont déclaré que le rapport sexuel était forcé. » (Henry 2008 : 9). Étant exclusivement consacrées aux hommes ayant des pratiques homosexuelles, ces enquêtes effectuées en Afrique n'offrent aucun élément de comparaison avec l'expérience éventuelle de rapports homosexuels imposés chez les hommes qui n'ont pas de pratiques homosexuelles. En France, en revanche, où de telles données existent, il a été montré que les homosexuels et bisexuels déclarent beaucoup plus souvent que les autres l'expérience de rapports contraints. Dans le cadre d'une enquête sur la sexualité des Français réalisée au début des années 1990, les homos- et bisexuels ont déclaré avoir subi des rapports sexuels imposés vingt fois plus souvent que les hétérosexuels (Messiah et Mouret-Fourme 1993) ; dans une nouvelle enquête réalisée quinze ans plus tard, presque cinq fois plus (Bajos et Bozon 2008). Chez les homosexuels et bisexuels concernés dans la première enquête, les rapports imposés ont eu lieu en moyenne six ans avant les premiers rapports sexuels volontaires.

Ces résultats invitent à questionner le lien éventuel entre l'initiation contrainte et l'adoption ultérieure de pratiques homosexuelles, en suggérant que les rapports imposés favorisent l'adoption ultérieure de pratiques homosexuelles, ou au minimum ne la découragent pas. Comment le comprendre?

Quelques enquêtes réalisées aux États-Unis sur les comportements sexuels en prison soulèvent la question de l'inclination homosexuelle en situation de contrainte, en récusant la distinction souvent faite à ce sujet entre homosexualité situationnelle et homosexualité véritable (Van Wormer 1984). Une étude menée auprès de neuf anciens détenus ayant eu des pratiques homosexuelles en prison, mais jamais précédemment, montre qu'après leur sortie, les agresseurs sont tous redevenus exclusivement hétérosexuels alors que les agressés ont poursuivi les pratiques homosexuelles (Sagarin 1976). Les hommes contraints au rapport homosexuel se sont donc durablement conformés au rôle que leurs agresseurs leur avaient assigné, au-delà du seul contexte de la prison. D'une manière éventuellement comparable, sans toutefois vouloir forcer l'analogie, on peut supposer que le rapport homosexuel contraint a pu favoriser chez certains jeunes Bamakois la formation d'un goût pour la sexualité entre hommes. Il faut d'ailleurs souligner que d'après les témoignages recueillis, désir et contrainte apparaissent souvent mêlés ; le viol constitue au fond la forme extrême d'un continuum de comportements sexuels qui ont en commun d'être incités par autrui, et dans le cadre desquels l'articulation entre la contrainte, le consentement et le désir varie fortement selon les cas.

Le fait qu'à Bamako, l'initiation homosexuelle soit souvent décrite comme impulsée, voire imposée de l'extérieur doit être compris en relation avec certaines caractéristiques du contexte local. Tout d'abord, la société malienne est très fortement hiérarchisée, notamment 
du point de vue générationnel. Les cadets sociaux sont soumis à l'autorité de leurs aînés et en particulier des membres de leur famille. Dans bien des cas, ils se doivent d'obtempérer face à la volonté des plus âgés. Ensuite, les relations sociales se trouvent structurées par le double critère de l'âge et du genre, produisant une très forte homosocialité. La force des relations nouées en particulier entre jeunes pairs générationnels s'exprime sous la forme d'une grande proximité des corps, à travers le fait de se tenir la main, s'asseoir l'un sur l'autre, s'enlacer, etc. ; autant de postures qui, en France, évoqueraient automatiquement un lien de nature érotique. À l'inverse, à Bamako, cette proximité corporelle tend plutôt à dés-érotiser les contacts physiques entre hommes, c'est-à-dire à rendre plus lointaine la probabilité du désir homo-érotique et la frontière entre sexuel et non sexuel. L'absence de signification sexuelle associée aux contacts physiques entre hommes montre que l'éventualité du rapport homosexuel ne hante pas les relations homosociales avec autant de prégnance que dans le contexte français. Pourtant, en même temps qu'elle témoigne du caractère souvent inconcevable du rapport homosexuel, la proximité permanente des corps masculins est aussi précisément ce qui, dans bien des cas, rend concrètement possible le passage à l'acte sexuel. En témoignent les scénarios récurrents de relations sexuelles entretenues par deux adolescents ou deux jeunes adultes occupant ordinairement le même lit - deux cousins ou deux amis par exemple -, sans d'ailleurs susciter le moindre soupçon dans l'entourage immédiat.

En France, l'existence de la catégorie homosexuelle incite, voire contraint, certains de ceux qui ont des pratiques avec des personnes de même sexe à s'y identifier. Elle structure la socialisation sexuelle (la socialisation genrée en même temps que la socialisation à la sexualité), fût-ce négativement, en favorisant la définition de soi, soit par opposition, soit par identification à cette catégorie. À Bamako, la socialisation sexuelle se caractérise par une moindre prégnance du modèle de référence - à la fois négatif et positif - de l'homosexualité comme catégorie sociale instituée, voire « publique ». Dès lors, dans un tel contexte, le désir homosexuel ne peut se formaliser sous la même forme que dans les sociétés où existe une telle catégorie endogène de représentation - dans le sens à la fois de conception socialement construite et d'image publique. Ainsi pouvons-nous expliquer qu'il soit rarement dit par les hommes concernés qu'ils avaient été attirés par les hommes avant de passer à l'acte, ou que certains affirment qu'ils ne savaient pas que l'homosexualité existait avant de se trouver confrontés à certaines sollicitations. Puisque l'homosexualité est censée ne pas exister au Mali, beaucoup d'hommes ne se posent pas la question de leur orientation sexuelle et souvent le goût homosexuel n'apparaît qu'après l'expérimentation, le passage à l'acte. N'étant pas suscité par la socialisation sexuelle ordinaire ou « primaire », le désir homosexuel est présenté dans les discours des intéressés comme naissant de l'initiation par un tiers, voire de la contrainte.

La conception de l'entrée dans la pratique homosexuelle comme processus de conversion par autrui apparaît à travers divers éléments de langage. On entend souvent les individus ayant des pratiques homosexuelles dire qu'ils ont été " mis dedans » par quelqu'un, l'expression « être dedans » indiquant dans ce cas le fait de faire partie des personnes qui ont des pratiques homosexuelles. La pratique homosexuelle est ici pensée comme nécessitant un initiateur, c'est-à-dire comme une forme de connaissance et de savoir-faire engendrée par un tiers. En témoignent aussi les termes français les plus courants pour évoquer l'homosexualité, « milieu » et « branché », qui désignent plus largement un groupe déterminé et le fait d'en 
faire partie, d'en détenir les clés, de répondre aux critères d'appartenance qui le définissent. Il arrive également d'entendre un homme qui a des pratiques homosexuelles dire au sujet d'autres personnes qu' « ils ne connaissent pas ça », c'est-à-dire qu'ils ne savent pas que cela existe et ne sont donc pas en mesure d'imaginer ce que peuvent faire sexuellement deux hommes entre eux. Enfin, certains désignent par le terme bambara « fàamu », qui signifie « comprendre » ou « compréhension », l'individu qui a connaissance de l'homosexualité à travers des personnes de son entourage mais n'en est pas lui-même un pratiquant avéré - ce qui rappelle étrangement le terme espagnol « entendido » que l'on retrouve aussi bien en Espagne que dans différents pays d'Amérique latine pour désigner une réalité proche, à savoir les hommes hétérosexuels qui ont des pratiques sexuelles avec des hommes efféminés. Ainsi, l'entrée dans la sexualité entre hommes est-elle souvent pensée et vécue comme un accès à un monde caché de pratiques réservées à des initiés, expliquant que beaucoup de ceux devenus « branchés » disent avoir été, la première fois, incités, voire plus ou moins contraints à la relation sexuelle par un tiers.

\section{... ou désir « naturel »?}

En appréhendant le mode d'entrée dans la sexualité de manière comparative, on constate donc que la définition sociale de l'homosexualité détermine la façon dont le désir s'exprime : en France, où la catégorie homosexuelle correspond à un modèle en référence auquel se structure tout parcours de socialisation sexuelle, beaucoup témoignent de leurs attirances homosexuelles avant le passage à l'acte (Lhomond 1997), ce qui est rare à Bamako, où le désir homosexuel se trouve plus souvent révélé suite à une initiation impulsée, voire imposée, par autrui. Toutefois, cette explication ne saurait suffire, en particulier parce que la contrainte semble ne pas toujours s'exercer au hasard. En effet, certains hommes décrivant une entrée dans la sexualité marquée par une incitation musclée expliquent présenter des caractéristiques qui ont pu favoriser le passage à l'acte de leur initiateur, tels que l'efféminement.

En effet, à Bamako, comme en France, certains jeunes garçons adoptent précocement des attitudes ou des goûts réputés féminins. Dans la plupart des cas, les rappels à l'ordre scandés par l'environnement social auront pour effet de les réorienter vers des rôles de genre plus conformes aux modèles prescrits. L'efféminement peut alors devenir une partie de la personnalité dont l'expression sera limitée à quelques contextes favorables. Plus rarement, il constitue une caractéristique permanente de l'individu. Mais il n'existe à Bamako aucune institution de l'inversion de genre qui pourrait s'apparenter aux catégories socialement reconnues d'hommes apparentés au genre opposé décrites ça et là dans la littérature anthropologique.

Selon certains des garçons interrogés, ce sont leurs attitudes féminines qui ont pu stimuler le comportement sexuel imposé par l'initiateur :

- Est-ce que tu peux nous expliquer un peu comment tu es arrivé à cette pratique homosexuelle?

- Je veux parler en majorité, quoi! J'étais comme ça depuis longtemps, mais je n'avais pas commencé la pratique. Je veux dire que moi je suis naturellement comme ça, comme une femme, quoi! Mais je n'avais pas commencé. Je peux dire que j'ai véritablement commencé cette année même. J'ai commencé parce qu'on m'a violé. J'étais ami avec quelqu'un, mais je ne savais pas qu'il faisait ce genre de chose. Il 
m'a violé en me faisant l'amour. J'avais quand même des modèles de femmes mais je n'avais pas encore commencé à faire la pratique. (Entretien 3, 20 ans)

Quand je commençais la pratique, j'étais encore très jeune (12 ans environ). J'apportais du manger pour ma grand-mère au jardin. Un beau jour j'ai rencontré un monsieur sur mon chemin qui amassait de l'herbe. Il m'a approché pour prendre satisfaction de moi. Il a éjaculé et m'a mouillé tout le corps de son sperme sans faire de pénétration anale au juste. Un bon moment après, j'ai découvert le domicile du monsieur avec mon camarade de classe qui fait la même résidence que lui. Dès lors je n'ai cessé de fréquenter le monsieur pour qu'il reprenne les mêmes choses.

- Pourquoi le monsieur a eu le courage de vous aborder en ce sens?

- C'est moi qui suis parti vers lui pour lui demander le passage car l'endroit était très herbacé. Bien avant, j'avais des caractéristiques féminines, je crois que c'est ce qui l'a surtout incité. (Entretien 6, 24 ans)

D’autres garçons évoquent différemment le rôle de leur efféminement, en suggérant non pas qu'il a incité des hommes à engager une relation sexuelle, mais qu'il a provoqué leur stigmatisation et que cette désignation extérieure de leur « homosexualité » est à l'origine de leur passage à l'acte :

- Comment êtes-vous venu à l'homosexualité?

- Depuis que j'étais petit, l'entourage me qualifiait d'homosexuel. À vrai dire j'avais la « déclaration », c'est-à-dire les signes féminins : la lenteur de la démarche, de la parole, la gesticulation. S'il arrivait que quelqu'un me qualifie, je pouvais l'insulter père et mère que je ne saurais pas prononcer maintenant. Cela date des années 19981999. Par mépris pour la qualification qu'ils m'attribuent, j'ai décidé par moi-même d'adhérer à la pratique contre leur gré.

- Pensez-vous que c'est seulement la raison?

- Oui. [...]

- Je n'arrive pas à comprendre comment les sabotages des gens vous ont poussé à cette pratique...

- À supposer que, quelqu'un de passage, qu'on lui dise "pédé ». C'est la même chose qu'on me disait.

- Est-ce ce qui vous a réellement poussé à cette pratique?

- Juste! C'est bien ça. (Entretien 11, 22 ans)

- Quelles sont les motivations liées à la pratique?

- Au delà de l'aspect matériel et du plaisir qu'on y trouve, on peut aussi retenir le caractère naturel de la pratique chez certains. Tel est mon cas par exemple. En effet, ayant une voix et un comportement à tendance beaucoup féminin, les gens me taxaient d'être du "milieu » alors que je ne l'avais pas encore intégré. Ils ne cessaient de me critiquer. C'est ainsi que j'ai lancé le défi à quiconque pour m'empêcher de faire cette pratique. Et c'est comme ça que j'ai commencé à vivre dans le «milieu ». Aujourd'hui personne ne peut me parler de ça ouvertement. (Entretien 28, 17 ans)

Ces nouveaux éléments permettent d'amender l'analyse qui précède, en ce qu'ils suggèrent fortement que l'efféminement qui caractérise certains jeunes hommes dits « déclarés » constitue l'un des facteurs qui déterminent leur entrée dans la sexualité par des effets d'assignation, qu'ils soient de l'ordre du rapport sexuel contraint ou de la stigmatisation provoquant par ricochet le passage à l'acte sexuel. Mais cet efféminement est aussi considéré par les intéressés comme le signe que leur attirance pour les personnes de même sexe est 
« naturelle ». Si la catégorie homosexuelle n'existe pas à Bamako avec la même prégnance qu'en France, certains expliquent néanmoins avoir été catégorisés comme faisant partie du « milieu » avant de considérer eux-mêmes qu'ils y appartenaient, et présentent a posteriori leurs attributs féminins comme annonciateurs d'un désir homosexuel.

Ces exemples montrent encore que la désignation extérieure porte moins sur le comportement sexuel effectif que sur la non-conformation des individus concernés aux normes de leur genre, en tant qu'elle annonce potentiellement une orientation du désir. De manière révélatrice, les deux termes les plus utilisés pour qualifier péjorativement les " homosexuels » masculins sont le mot wolof " góor-jigéen », qui se traduit littéralement par « homme-femme », et le mot bambara «sa», qui signifie « serpent», en référence au mouvement ondulatoire de l'animal, en ce qu'il évoque une démarche féminine. Ce dernier terme est utilisé plus largement comme injure en direction des hommes jugés insuffisamment masculins et témoigne donc à la fois du stigmate que suppose la non-conformation de genre et du fait que le comportement homosexuel est conçu principalement comme une modalité du genre. Mais ces différents éléments indiquent aussi que le comportement homosexuel, à travers une lecture comme inversion de genre impliquant donc une inversion du désir, peut être considéré comme "naturel » dans le contexte bamakois, tant par les personnes directement concernées que par les autres.

Ainsi, à travers les différents propos relevés ici, deux conceptions majeures du comportement homosexuel s'expriment, l'une considérant qu'il renvoie à un savoir-faire nécessitant une initiation, l'autre selon laquelle certains individus y seraient naturellement disposés par leur apparentement au genre opposé, ces deux conceptions n'étant évidemment pas exclusives l'une de l'autre. En même temps que deux conceptions du désir homosexuel, ce sont aussi deux populations que l'on voit se dessiner, celle des hommes efféminés et celles des hommes conformes aux normes de la masculinité - les deux découpages étant partiellement superposables. Cette dichotomie implique un rapport différencié au stigmate : en fonction du degré d'identification extérieure de la personne comme ayant des pratiques homosexuelles, notamment selon sa conformation ou non aux normes de genre, l'exposition au risque de stigmatisation et sa gestion peuvent varier. Dans tous les cas cependant, chacun entend éviter le sentiment de honte qu'induit l'adoption de comportements socialement considérés comme inconvenants, dont font partie les pratiques homosexuelles.

\section{Se soustraire à la honte}

Dans une série d'articles connus pour les critiques qu'ils ont inspirées, John C. Caldwell et ses collaborateurs ont voulu caractériser la « sexualité africaine » par opposition au système eurasien, en postulant notamment l'absence de « culpabilité profonde » (deep guilt) dans la conception africaine de la sexualité (Caldwell et al. 1989), ce point faisant partie de ceux qui ont été contestés, même s'il n'est pas le principal. Dans le cadre de ces échanges, ni l'équipe de Caldwell, ni la majorité des critiques n'ont évoqué les comportements homosexuels, conformément au silence plus général des chercheurs sur le sujet jusqu'au début des années 2000 (Epprecht 2008 ; Broqua 2009b). Pourtant, l'absence supposée de culpabilité liée à la sexualité ne devrait-elle pas être logiquement jugée favorable à l'existence de comportements homosexuels ? Il ne faudrait pas que les propositions contestables de l'équipe de Caldwell entraînent le rejet de toute interrogation sur le sujet, car la question du lien éventuel entre culpabilité et sexualité en général, ou entre culpabilité et pratiques homosexuelles en 
particulier, mérite certainement d'être posée. Pour y répondre, il peut être utile de revenir à la distinction classiquement établie entre la culpabilité et la honte. Fréquente en psychologie, cette distinction a d'abord marqué la littérature anthropologique (Benedict 1946 ; Mead 1950). Dans un ouvrage phare de l'école culturaliste, où sont comparés le Japon et les États-Unis, Ruth Benedict (1946) distingue «cultures de la culpabilité » (guilt cultures) et «cultures de la honte » (shame cultures). Dans les premières (ici les États-Unis), les conduites sont dictées par une logique intrapsychique supposant l'intériorisation de la faute et l'examen de conscience, tandis que dans les secondes (ici le Japon), c'est la crainte du regard des autres et des sanctions externes qui régule les comportements. Cette distinction a bien sûr été fortement critiquée, à l'instar du principe même fondant la méthode culturaliste mise en œuvre par Ruth Benedict, pour son généralisme et (donc) son réductionnisme. Toutefois, la distinction entre honte et culpabilité ne doit pas être entièrement rejetée ; sa portée explicative s'avère non négligeable dans le cas d'une approche comparée des pratiques homosexuelles.

Dans les pays d'Amérique du Nord ou d'Europe, en matière d'orientation homosexuelle, la honte est souvent conçue et désignée comme le sentiment à combattre par excellence (Hillier et Harrison 2004). Les mouvements homosexuels lui ont opposé l'injonction à la fierté (pride). En revanche, la culpabilité, dont la puissance régulatrice est au moins aussi forte, se trouve rarement mise en cause. Lorsqu'elle est évoquée, elle peut même être présentée comme bénéfique ; un psychiatre et psychanalyste écrit ainsi : " la honte pour une faute publiquement découverte est beaucoup plus gravement redoutée que la culpabilité attachée à des fautes qui peuvent rester secrètes. La culpabilité est une forme d'intégration sociale alors que la honte est une désintégration. » (Hefez 2003 : 151).

Au Mali, comme dans bien d'autres pays africains, la honte (màlo en langue bambara) constitue un puissant régulateur social, en ce qu'elle représente une menace contre l'honneur (dànbe) et doit donc être évitée par tous les moyens possibles (Bouju 2004). Dans ce contexte, le comportement homosexuel est bien plus fortement régulé par la honte que par la culpabilité. Un homme qui n'éprouve pas de culpabilité manifeste à adopter des comportements homosexuels peut dire avoir honte de tout geste démonstratif en public avec son partenaire par exemple. Alors qu'en France, les comportements sexuels sont conditionnés avant tout par l'incorporation des normes de la morale chrétienne et des sciences médicales ou psychologiques qui ont progressivement façonné la définition sociale des catégories sexuelles, ce sont davantage les conditions sociales de l'exercice de la sexualité, en particulier celles relatives à l'évitement de la honte, qui s'avèrent contraignantes à Bamako.

Toutefois, bien que la honte domine incontestablement dans le contexte malien, la culpabilité n'y est pas inexistante. Pour déceler le sentiment de culpabilité qui peut être associé aux pratiques ou aux désirs homosexuels, il peut être utile de s'intéresser non pas tant aux individus qui s'adonnent à de telles pratiques qu'à ceux qui sont tentés mais s'interdisent de passer à l'acte, à l'instar de Moussa par exemple. Celui-ci réside à proximité d'un maquis de Bamako fréquenté par de nombreuses personnes ayant des pratiques homosexuelles, dans lequel il se rend parfois. Ayant été sollicité par certains hommes, il en vient progressivement à s'interroger sur son désir d'expérimenter la pratique homosexuelle. Sans jamais oser tenter l'expérience, il éprouve (et exprime) continuellement la tension psychique que ce désir lui inspire. Moussa est un fervent musulman et lorsqu'il évoque cette impossibilité de passer à l'acte en dépit de son désir, il l'explique généralement par son 
engagement religieux. Ce seul exemple suffit à montrer que la régulation des comportements homosexuels ne relève pas exclusivement des stratégies visant l'évitement de la honte, mais aussi d'un sentiment de culpabilité, puisque certains s'interdisent des pratiques sexuelles qui pourraient avoir lieu sans que quiconque en ait connaissance. Cette culpabilité découle en grande partie de l'intériorisation d'une conception du rapport homosexuel comme péché et de sa condamnation par l'Islam, sachant que les références à ce comportement ne sont pas rares lors des prêches à Bamako. Aussi bien, pendant le mois de carême, nombreux sont les pratiquants qui s'abstiennent de toute relation sexuelle en général, et homosexuelle en particulier. Si la sexualité se trouvait exclusivement régulée par le risque de honte que peut engendrer le regard de l'autre, pourquoi ici encore certains s'interdiraient-ils des comportements sexuels pouvant s'accomplir à l'abri des regards ? L'intégration et le respect des normes de la sexualité dictées par l'Islam renforcent chez certains l'examen de conscience individuel, et nourrissent en cela un sentiment de culpabilité qui se superpose au sens de la honte.

Mais tous les hommes concernés entretiennent-ils le même rapport à la honte ? Dans un article sur les pratiques homosexuelles au Nicaragua, Roger N. Lancaster montre qu'une distinction nette s'opère entre hommes " actifs » et hommes " passifs » dans la relation sexuelle : les premiers ne mettent pas leur honneur en danger tandis que les seconds sont susceptibles de honte (Lancaster 1988). À Bamako, dans les conceptions comme dans les pratiques de l'homosexualité, il existe aussi une séparation entre les rôles « actifs » et " passifs », qui renvoie en partie à une distinction entre les hommes efféminés et les autres. Mais cette distinction est moins clivante qu'au Nicaragua du point de vue de la respectabilité sociale. Même lorsqu'ils se conforment aux normes dominantes de la masculinité, les hommes qui ont des pratiques homosexuelles dans un rôle « actif » encourent le risque d'être stigmatisés si cela se sait. Contrairement au tableau dépeint à propos du Nicaragua, le partenaire « actif » n'échappe pas automatiquement à la honte à Bamako, où toute assimilation à un comportement homosexuel est potentiellement stigmatisée.

Soucieux de contrer la menace sociale de la honte, les hommes qui ont des pratiques homosexuelles se montrent attachés à la sutura. La notion de sutura, surtout connue au Sénégal en langue wolof, mais qui existe aussi en langue bambara, désigne la discrétion, le comportement susceptible de préserver la réputation et le respect de la vie intime. Ici, le meilleur moyen d'échapper à la honte est le silence ou le mensonge qui, plus généralement, sont constamment mobilisés dans les relations sociales. Alors qu'en France, le sentiment de culpabilité associé à l'homosexualité découle notamment du mensonge que beaucoup s'imposent sur leurs pratiques, en tant qu'il est contraire à la morale (religieuse ou ordinaire), au Mali le mensonge correspond au contraire à l'un des principaux outils permettant de se soustraire à la honte. Ici, l'exposition au risque de honte n'est pas distribuée selon le rôle que les individus occupent dans la relation sexuelle, mais bien selon leur aptitude à ne pas faire montre de leurs pratiques ou inclinaisons homosexuelles.

\section{Savoir taire le désir homosexuel}

À Bamako, les personnes qui ont des pratiques homosexuelles s'emploient à le dissimuler, comme l'indique bien le vocabulaire de l'homosexualité qui recourt dans ce même but à des termes non explicites et non spécifiques, tels que «branché » ou « milieu ». Si des stratégies sont développées afin de maintenir le secret sur ces pratiques, c'est que l'hostilité 
à l'égard de l'homosexualité est fort prégnante. Parmi les plus enclins à exprimer une telle hostilité, certains Bamakois travaillant en contact avec les "toubabs » (Blancs) sont en bonne place, pour des raisons qui procèdent précisément de leur proximité ambiguë avec les visiteurs occidentaux. En même temps, les hommes qui ont des pratiques homosexuelles, y compris lorsqu'ils sont aisément identifiables comme tels par leur seule apparence, ne sont pas forcément l'objet de remarques ou d'injures frontales. À ce sujet comme sur bien d'autres points, chacun fait vis-à-vis des autres comme s'il ne savait pas, tant que rien n'est verbalisé par l'intéressé. Les personnes concernées peuvent bénéficier d'une relative indulgence dès lors qu'elles font jouer cette règle du silence imposée par les convenances sociales qui, en principe, interdisent de s'étendre sur les éléments discréditables de la vie privée d'un individu en sa présence. Afin d'exemplifier ce processus, je présenterai la situation concrète d'un jeune Bamakois.

Issa vit avec sa famille dans un quartier populaire de la ville, depuis sa naissance à la fin des années 1970. Après avoir fait l'expérience de relations sexuelles avec quelques jeunes femmes, maliennes et françaises, il rencontre au début des années 2000 un Français résidant au Mali, avec lequel il découvre la sexualité entre hommes. Quelques mois plus tard, il fait la connaissance de David, un autre Français, avec lequel il décide d'entretenir une relation suivie. Placé au contact de toubabs dans le cadre de son activité professionnelle, il se trouve immergé dans un milieu d'individus qui vivent du même travail et dont beaucoup résident dans son quartier. Tous le respectent, en partie en raison du fait qu'il leur procure régulièrement du travail. Issa est un noctambule et sort presque chaque soir dans les maquis les plus animés de la ville, dont certains sont fréquentés à la fois par des hommes ayant des pratiques homosexuelles et par ces jeunes hommes qui travaillent avec des toubabs. Son nouveau compagnon l'accompagne le plus souvent et, comme il est de coutume au sein d'un couple dont l'un des partenaires est plus fortuné que l'autre, il prend en charge pour Issa les frais de chaque sortie. Ainsi, cette relation est rapidement connue de ceux qui ont l'œil exercé. C'est qu'à Bamako, le contrôle social s'exerce sans relâche ; afin de garantir le secret sur ses pratiques sexuelles, il est nécessaire de déployer des trésors d'ingéniosité en matière de camouflage, de non-dit ou de faux-semblant, et le savoir-faire en la matière excelle chez beaucoup. La particularité du couple que forment Issa et David est d'être confronté, lors de ses sorties, à la fois aux hommes ayant des pratiques homosexuelles qui les considèrent comme des «pairs ", et aux jeunes hommes travaillant avec des toubabs que connaît très bien Issa. David se demande durant plusieurs mois si ces derniers comprennent la nature du lien qui l'unit à son compagnon. Lorsqu'il interroge Issa à ce sujet, ce dernier lui répond avec assurance qu'ils ne savent rien. Pourtant, Issa informe David à plusieurs reprises du fait que certains d'entre eux ont fait part à son grand frère de leurs soupçons, voire de leurs certitudes quand à l'homosexualité de David, le « si proche ami d'Issa ». Au fil du temps, les liens entre nos deux protagonistes se distendent. Au moment de la saison froide, qui est aussi la saison la plus touristique, David sort de plus en plus souvent sans Issa, trop accaparé par son travail. À différentes reprises, il se trouve alors confronté à des remarques ou des injures proférées par des collègues d'Issa, qui comportent des allusions évidentes à leur relation. Le lendemain de l'incident le plus virulent, David le raconte à Issa qui lui répond que ses agresseurs ne se seraient jamais permis de tels comportements en sa présence. Ce dernier décide d'intervenir avec son grand frère auprès des agresseurs qu'ils connaissent bien, lesquels présentent finalement des excuses. David découvre ainsi que les proches 
d'Issa avaient compris probablement depuis longtemps la nature du lien l'unissant à son ami français, mais qu'ils ne pouvaient se permettre d'y faire allusion en sa présence.

Cet exemple montre de manière claire qu'il ne suffit pas que les pratiques homosexuelles d'un individu soient connues pour être dénoncées ; le silence d'Issa sur leur existence remplit une fonction performative. Comme l'écrit Raymond Jamous au sujet du monde méditerranéen : "Si le mensonge met l'autre à distance, c'est moins pour l'empêcher de connaître votre secret que pour l'empêcher de le révéler, d'en parler en public devant vous. » (Jamous 1993). En revanche, les conditions sociales ne sont pas réunies pour que David échappe à une agression qui est ici possible contre un toubab " isolé », alors qu'elle ne l'aurait pas été envers un Bamakois ordinairement habile. La logique mise en exergue par cet exemple renvoie plus largement à la fonction sociale du silence et du mensonge au Mali (Hoffman 1998 ; Jansen 2001), bien illustrée par le terme bambara gundo qui, généralement traduit par « secret », désigne aussi une réalité qui est connue de tous mais dont personne ne doit parler.

Le rôle central du secret, du silence et du mensonge dans la gestion du risque de stigmate lié aux pratiques homosexuelles, à Bamako comme dans d'autres contextes africains (Courtray 1998 ; Teunis 2001), ne peut manquer d'apparaître fortement contradictoire avec la conception dominante de l'accomplissement homosexuel aux États-Unis ou en France par exemple (Broqua et de Busscher 2003). Depuis plus d'un siècle, l'orientation sexuelle y est soumise à une véritable épreuve de vérité, permettant de satisfaire la "volonté de savoir » (Foucault 1976) au moyen de technologies confessionnelles tout d'abord mises en œuvre par la religion, la médecine, la psychiatrie ou la psychanalyse, et relayées aujourd'hui par les médias et les nouvelles techniques d'information et de communication. Le coming out, par lequel est désignée la révélation à autrui de sa propre homosexualité, est devenu une étape incontournable de la carrière homosexuelle, prescrite par les homosexuels eux-mêmes depuis l'apparition des mouvements dits de libération, en même temps que par l'ensemble de la société (Cain 1991 ; Herdt et Boxer 1993). Bien entendu, cette incitation au discours et au dévoilement ne suffit pas à homogénéiser les modes de vie ou les formes identitaires, mais elle impose un modèle dominant en fonction duquel se constitue tout positionnement en la matière.

Pour beaucoup d'hommes ayant des pratiques homosexuelles à Bamako, l'idée de coming out, telle que définie par son acception la plus récente, n'a pas de sens. Cette volonté de faire savoir est en effet parfaitement contraire au principe de discrétion, et même de brouillage des pistes, à propos de la vie intime, qui guide plus généralement le comportement de chacun à Bamako. Cela ne signifie pas que la «volonté de savoir » n'y existe pas, mais qu'elle ne peut y être satisfaite par les mêmes techniques qui la servent en France ou aux États-Unis. Contrairement aux pratiques confessionnelles chrétiennes qui cherchent à délier les langues, le contexte musulman de Bamako incite plutôt au silence. Face aux interdits de l'Islam, en matière d'homosexualité comme dans le cas d'autres comportements proscrits, le mensonge représente une ressource inépuisable.

Dans le cadre de l'idéologie du dévoilement qui s'impose en France ou aux ÉtatsUnis, dire le désir homosexuel est aussi le consacrer, c'est atteindre le stade ultime de l'accomplissement, celui de la libération. Contestant « l'hypothèse répressive » soutenue par les mouvements de libération, Michel Foucault a bien montré que le fait de dire sa sexualité ou son désir, c'est d'abord répondre à l'incitation au discours (Foucault 1976). 
Mais il faudrait ajouter que dans ces pays occidentaux, c'est aussi se libérer du sentiment de culpabilité que provoque le silence sur le fait de se considérer homosexuel. À Bamako, le sentiment de culpabilité associé à la pratique homosexuelle est moins prégnant, comme j'ai voulu le montrer, et surtout, lorsqu'il existe, il ne peut en aucune manière trouver le soulagement par la confession.

L'expression coming out n'a cependant pas toujours été définie par la signification étroite que nous lui connaissons aujourd'hui ; elle a connu une évolution sémantique. Autrefois, elle désignait non pas le fait de révéler son homosexualité auprès de son entourage, mais l'intégration au monde homosexuel (Chauncey 1994). Dans ce sens plus ancien, elle peut aider à décrire le processus de socialisation homosexuelle d'une partie des hommes rencontrés à Bamako, et des modes d'articulation possibles entre désir homosexuel et constructions identitaires.

\section{Sentiments d'appartenance et trajectoires identitaires}

Le silence entourant le désir homosexuel s'accompagne plus largement, dans la majorité des cas, d'une conformation au modèle dominant de la conjugalité hétérosexuelle et de la reproduction. L'expérience homosexuelle est presque toujours une expérience parallèle, qui peut occuper une place importante dans la vie de l'individu, mais ne se substitue pas à la carrière hétérosexuelle et aux rôles d'époux, de père, de chef de famille que suppose ordinairement le statut masculin. Pour les hommes concernés, le meilleur moyen de ne pas inspirer de soupçons sur le fait d'avoir des pratiques homosexuelles est d'être officiellement engagé dans une relation de couple avec une femme (au minimum); à partir d'un certain âge (la trentaine environ), ne pas se marier est perçu comme anormal, à plus forte raison si l'on gagne sa vie. Mais cette conformation à la conjugalité hétérosexuelle n'est pas nécessairement une couverture, elle répond souvent à une aspiration que la pratique ou le désir homosexuels ne concurrencent pas. Dans le contexte bamakois, les expériences homosexuelles peuvent être vécues intensément sans empêcher que soit parallèlement respecté l'ordre dominant de la parenté et de la reproduction.

Le fait de taire le désir homosexuel ou celui de se conformer à la conjugalité hétérosexuelle n'interdisent pas à une partie au moins des hommes ayant des pratiques homosexuelles d'éprouver le sentiment d'appartenir à un groupe formé par le partage d'un désir sexuel commun. Dans les propos des individus concernés, entendus dans la vie quotidienne ou lors des entretiens, affleurent sans cesse différents signes d'identification à un groupe. Le seul fait de nommer l'homosexualité par les termes « milieu » et «branché » signale une conscience collective. Dans les entretiens, on constate clairement un sentiment d'appartenance communautaire, que renforcent le silence et le secret partagés, ainsi que la capacité des individus concernés à identifier leurs pairs, à l'inverse du plus grand nombre, ce que l'un d'eux exprime ainsi : «C'est comme une association de sorciers, dès qu'on se voit, on se reconnaît. » (Entretien 7, 16 ans). C'est aussi que parallèlement au silence maintenu vis-à-vis de l'extérieur, la circulation, parmi les personnes concernées, d'informations sur les pratiques homosexuelles des uns ou des autres, crée les conditions d'existence d'un vaste réseau d'interconnaissance.

Dans de nombreux pays du monde, la vie homosexuelle urbaine se caractérise par l'existence de lieux de rencontre publics, extérieurs ou fermés, où s'exerce une sexualité dite « anonyme », c'est-à-dire entre partenaires qui ne se connaissent pas et n'échangent 
parfois aucun mot, dessinant les contours d'une « communauté silencieuse » (Delph 1978). Il n'existe pas d'espace de ce type à Bamako, à l'exception d'un lieu de drague extérieur assez peu fréquenté. Parce que les espaces de rencontre spécifiques sont rares et que les risques de dévoilement sont importants dans la vie courante, les hommes qui ont des pratiques homosexuelles ont pour habitude de se présenter mutuellement leurs connaissances, dans le but de nouer des relations sexuelles ou amicales. Ce faisant, ils tissent un réseau social alimenté par la loi du silence que chacun s'efforce de respecter sur ses propres pratiques, tout en faisant en sorte qu'elle ne soit pas brisée par les autres. Ces mises en relation limitent l'isolement et favorisent la solidarité, mais elles renforcent en même temps le contrôle social qui s'exerce au sein même de ce monde parallèle.

Depuis quelques années, ce réseau est alimenté par les rencontres qui s'effectuent au moyen d'internet. Différents sites gratuits sont utilisés par les hommes en quête de relations homosexuelles à Bamako. Progressivement, au fil des rencontres rendues possibles par ces sites, leurs usagers ont formé un nouveau réseau d'interconnaissance disséminé dans toute la ville, qui s'est greffé au premier. L'une des caractéristiques notables de ces nouveaux modes de rencontre, en particulier dans une perspective comparée, c'est ici encore la rareté des pratiques de « sexualité anonyme », que l'usage d'internet favorise pourtant fortement dans d'autres régions du monde. Alors que souvent, les individus qui utilisent internet à Bamako ne sont pas les mêmes que ceux qui sont déjà insérés dans des formes de sociabilité homosexuelle, ils vont progressivement intégrer le « milieu » suite à leur rencontre avec d'autres usagers des sites qui en font déjà partie, à travers par exemple la fréquentation de lieux publics spécifiques, tels que certains maquis.

Ainsi, en raison de l'information qui circule et des rencontres en chaîne qui s'effectuent dans la vie courante, avec ou sans le recours à internet, l'anonymat entre hommes ayant des pratiques homosexuelles est très rare, à l'inverse de la situation des métropoles européennes ou américaines. L'existence du réseau social formé par ces relations dépend en premier lieu du silence que ceux qui le composent maintiennent sur leur sexualité. Le fait que chacun soit informé des pratiques homosexuelles des autres ne permet pas seulement l'identification de « semblables » avec lesquels s'associer ou nouer des relations de différentes natures, mais aussi de disposer d'informations qui pousseront chacun à taire celles dont il dispose sur l'autre. Chacun obtient le respect du silence de l'autre par le fait de connaître ses pratiques, sur la base d'un contrat tacite selon lequel si l'un parle, l'autre le fera aussi. Si cela fonctionne le plus souvent, il arrive cependant que certains se livrent à des actes de chantage ou à des révélations gênantes.

Loin d'être homogène, en dépit du sentiment d'appartenance commune, la population des hommes ayant des pratiques homosexuelles se trouve clivée en deux principaux groupes, comme nous l'avons vu plus haut : ceux qui ne sont pas identifiables à l'œil nu et ceux qui sont parfois qualifiés de "déclarés », c'est-à-dire dont l'apparence efféminée à valeur d'affirmation publique de l'orientation sexuelle. Cette division rappelle très fortement celle entre groupes d' " homosexuels secrets » et d' " homosexuels déclarés » qui structurait la « communauté homosexuelle» de Montréal dans les années 1950 (Leznoff et Westley 1956). À Bamako, comme dans le contexte étudié par Leznoff et Westley, les hommes qui ne sont pas repérables craignent fortement l'assimilation à ceux qui le sont, et évitent soigneusement leur compagnie afin de n'encourir aucun risque de dévoilement. Le fait qu'il existe ainsi deux catégories d'hommes dont les relations oscillent entre défiance et évitement, à la fois 
atteste du sentiment de constituer une communauté globale (ce que déplorent les uns et soulignent les autres) et rend impossible son développement unifié.

Comme dans d'autres capitales du monde, la socialisation homosexuelle à Bamako s'effectue donc chez beaucoup en deux temps : celui de la socialisation primaire, principalement hétérocentrée, puis celui de l'intégration à un réseau d'interconnaissance parallèle. Mais ici, la phase de socialisation sexuelle secondaire n'implique généralement pas de divulgation publique de l'orientation sexuelle. Contrairement aux scénarios majoritairement prescrits (sinon suivis) en France ou aux États-Unis, l'expérience des Bamakois montre que le désir homosexuel peut être vécu sans que s'impose la tentation de choisir un mode de vie alternatif dans le cadre duquel s'exprimerait une vérité du sujet définie par sa seule sexualité. Si les trajectoires identitaires des hommes ayant des pratiques homosexuelles sont façonnées par cette expérience de l'homosexualité parallèle, la socialisation secondaire que cette expérience suppose parfois s'accomplit le plus souvent sans rupture biographique, notamment du point de vue familial. Autrement dit, alors qu'en France, chez les homosexuels, la phase de socialisation secondaire s'accompagne souvent d'un certain nombre de changements déménagement vers une plus grande ville, distension des liens avec l'entourage familial ou amical initial, etc. - à Bamako, l'intégration au « milieu » s'effectue le plus souvent de manière parallèle voire superposée (mais invisible) au maintien des liens familiaux, amicaux ou communautaires forgés au cours de la socialisation primaire.

Certains hommes n'entendent cependant pas suivre cette voie de l'homosexualité vécue parallèlement à la conjugalité hétérosexuelle ou limitée à l'inversion de genre. Une distance est alors prise à l'égard du modèle local des identités sexuelles minoritaires et du principe dominant qui détache la vérité du sujet de sa sexualité, pour lui substituer une conception de l'authenticité de soi où les pratiques homosexuelles trouvent une place. Certains hommes qui quittent le Mali pour l'Europe, l'Amérique du Nord ou d'autres pays plus proches, provisoirement ou plus durablement, vont parfois suivre une trajectoire de socialisation homosexuelle plus fortement construite en référence à des modèles extérieurs. Par exemple, Alassan, alors qu'il s'apprête à quitter Bamako pour un pays du Maghreb afin de s'y installer avec son compagnon français et d'y poursuivre ses études, m'explique que l'on ne peut compter que sur soi pour s'extraire des contraintes qui pèsent sur l'individu du point de vue de la trajectoire sexuelle ou conjugale. Dernier enfant de sa fratrie, il a très tôt œuvré à conquérir son autonomie pour ne pas se trouver confiné par l'emprise de sa famille et l'autorité de ses aîné-e-s, puisqu'il ne souhaitait ni se marier ni avoir d'enfant, ce qui est très rare à Bamako. Lorsque ses sœurs lui demandent quand il va se marier et faire des enfants, il leur répond qu'elles ont déjà elles-mêmes beaucoup d'enfants, que ce sont aussi « ses » enfants. Il leur dit qu'il veut simplement réaliser son rêve, et lorsqu'elles lui demandent quel est ce rêve, il leur répond que cela ne les regarde pas. Au sujet de sa lecture en cours d'un ouvrage sur l'homosexualité en France qu'il m'a demandé de lui procurer, il me dit que cela l'intéresse car cela lui apporte beaucoup d'informations sur l'histoire, avant d'ajouter : « Je ne veux pas être étranger dans ma propre vie. »

$\mathrm{Si}$, dans le contexte bamakois, le désir homosexuel contribue moins à définir la vérité du sujet que dans d'autres contextes, il apparaît à l'issue de ce texte qu'il n'est pas sans effet sur les constructions identitaires, individuelles et collectives. L'un des signes en est 
ce sentiment d'appartenance qui relie bien des hommes ayant des pratiques homosexuelles. Le débat houleux qui a opposé approches constructionnistes et essentialistes de l'histoire de l'homosexualité portait notamment sur ce point : les tenants de la première approche reprochaient aux seconds un anachronisme lorsqu'ils évoquaient des groupes plutôt que des pratiques à propos des époques au cours desquelles n'existait pas de catégorie «homosexuelle» socialement instituée. Il ne faudrait pas laisser cet antagonisme se rejouer non plus sur une échelle diachronique mais sur celle de la géographie culturelle, en considérant que dans un contexte où, comme à Bamako, la catégorie sociale de l'homosexualité ne s'affirme pas sous une forme instituée ni publique, il n'existerait que des comportements homosexuels et aucune identité associée. Bien que la catégorie homosexuelle y soit peu prégnante et souvent rejetée comme étrangère aux mœurs locales, il existe un sentiment communautaire et donc une production identitaire qui découlent non seulement de la pratique mais aussi du désir homosexuel. Car si le sentiment d'appartenance collective dont témoignent les « branchés » bamakois n'est pas exactement assimilable à l'identité homosexuelle des capitales d'Europe ou d'Amérique du Nord, il n'est pas réductible au seul partage d'une même pratique sexuelle mais se fonde aussi sur l'idée d'un désir sexuel commun. En se focalisant sur une distinction entre pratiques et identités, les tenants de l'approche constructionniste ont négligé cet élément qui structure pourtant les catégories de pensée de la sexualité et mérite en cela toute l'attention des sciences sociales. En dépit des réserves de Michel Foucault concernant cette notion, le désir n'est pas seulement un instrument servant la sujétion des individus, mais aussi un outil de subjectivation dont certains se saisissent pour négocier la définition de leur identité sociale, à Bamako comme ailleurs.

\section{Références citées}

BAjos, Nathalie et Michel Bozon, 2008. «Les agressions sexuelles en France : résignation, réprobation, révolte », in Nathalie Bajos, Michel Bozon et Nathalie Beltzer (éds), Enquête sur la sexualité en France : pratiques, genre et santé. Paris : La Découverte, p. 381-407.

Benedict, Ruth, 1987 [1946]. Le chrysanthème et le sabre. Arles : Philippe Picquier.

Bouju, Jacky (éd.), 2004. Les incivilités de la société civile : espace public urbain, société civile et gouvernance communale à Bobo-Dioulasso et Bamako. Paris : rapport GEMDEV-ISTED.

Broqua, Christophe,

2009a. "Sur les rétributions des pratiques homosexuelles à Bamako », Canadian Journal of African Studies/Revue canadienne des études africaines, 43 (1), p. 60-82.

2009b. «Une découverte scientifique récente : l’homosexualité en Afrique », in Jean-Philippe Cazier (éd.), L'objet homosexuel : études, constructions, critiques. Mons : Sils Maria, p. 87-97.

Broqua, Christophe et Pierre-Olivier De Busscher, 2003. « La crise de la normalisation : expérience et condition sociales de l'homosexualité en France ", in Christophe Broqua, France Lert et Yves Souteyrand (éds), Homosexualités au temps du sida : tensions sociales et identitaires. Paris : ANRS, p. 19-33.

CAIN, Roy, 1991. «Disclosure and secrecy among gay men in the United States and Canada : a shift in views », Journal of the History of Sexuality, 2 (1), p. 25-45.

Caldwell, John C., Caldwell, Pat et Pat Quiggin, 1989. «The social context of AIDS in Sub-Saharan Africa », Population and Development Review, 15 (2), p. 185-134.

Carrier, Joseph et Stephen O. Murray, 1998. « Woman-woman marriage in Africa », in Stephen O. Murray et Will Roscoe (éds), Boy wives and female husbands : studies of African homosexualities, London-New York : St Martin's Press, p. 255-266. 


\section{Christophe BroquA}

Chauncey, George, 2003 [1994]. Gay New York, 1890-1940. Paris : Fayard.

Courtray, François, 1998. «La loi du silence : de l'homosexualité en milieu urbain au Maroc », Gradhiva, 23, p. 109-119.

Delph, Edward W., 1978. The silent community : public homosexual encounters. London : Sage.

Dennis, Jeffery P., 2000. «From Arcadia to Utopia : manipulating same-sex desire in ethnographic texts », Journal of Contemporary Ethnography, 29 (5), p. 618-640.

Epprecht, Marc, 2008. Heterosexual Africa? The history of an idea from the age of exploration to the age of AIDS. Athens : Ohio University Press.

Epstein, Steven, 1991. «Sexuality and identity : the contribution of object relations theory to a constructionist sociology », Theory and Society, 20 (6), p. 825-873.

FoucAult, Michel,

1976. La volonté de savoir. Paris : Gallimard.

1996. « Le gai savoir » (entretien), La revue h, 2, p. 42-54.

GAUdiO, Rudolf Pell, 2009. Allah made us : sexual outlaws in an islamic African city. Chichester : Wiley-Blackwell.

Greenberg, David F., 1988. The construction of homosexuality. Chicago : University of Chicago Press.

Gueboguo, Charles, 2006. La question homosexuelle en Afrique : le cas du Cameroun. Paris : L'Harmattan.

Gueye, Mouhamadou, Sarah CAStle et Mamadou Kani Konaté, 2001. «Timing of first intercourse among Malian adolescents : implications for contraceptive use », International Family Planning Perspectives, 27 (2), p. 56-62.

Harries, Patrick, 1990. «La symbolique du sexe : l'identité culturelle au début de l'exploitation des mines d'or du Witwatersrand », Cahiers d'études africaines, 120, p. 451-474.

Hefez, Serge, 2003. « Adolescence et homophobie : regards d'un clinicien », in Christophe Broqua, France Lert et Yves Souteyrand (éds), Homosexualités au temps du sida : tensions sociales et identitaires. Paris : ANRS, p. 147-156.

HENRY, Émilie, 2008. Identités, pratiques sexuelles et risques en matière de VIH/sida chez les hommes ayant des relations sexuelles avec d'autres hommes dans la ville de Douala (Cameroun). Mémoire DU Santé publique et promotion de la santé, Université Henri Poincaré, Nancy 1.

Herdt, Gilbert, 2004. « Sexual development, social oppression, and local culture », Sexuality Research and Social Policy, 1 (1), p. 39-62.

Herdt, Gilbert et Andrew Boxer, 1993. Children of horizons : how gay and lesbian youth are forging a new way out of the closet. Boston : Beacon Press.

Herdt, Gilbert et Robert J. Stoller, 1990. Intimate communications : erotics and the study of culture. New York : Columbia University Press.

Hillier, Lynne et Lyn Harrison, 2004. « Homophobia and the production of shame : young people and same sex attraction », Culture, Health and Sexuality, 6 (1), p. 79-94.

Hocquenghem, Guy, 1972. Le désir homosexuel. Paris : Éditions Universitaires.

Hoffman, Barbara G., 1998. "Secrets and lies : context, meaning, and agency in Mande », Cahiers d'études africaines, 149, p. 85-102.

JAMOUS, Raymond, 1993. « Mensonge, violence et silence dans le monde méditerranéen », Terrain, 21, p. 97-110.

JANSEn, Jan, 2001. Epopée, histoire, société : le cas de Soundjata, Mali et Guinée. Paris : Karthala.

LANCASTER, Roger N., 1988. « Subjet honor and object shame : the construction of male homosexuality and stigma in Nicaragua », Ethnology, 27 (2), p. 111-125. 
Leznoff, Maurice et William A. Westley, 1956. "The homosexual community », Social Problems, 3 (4), p. 257-263.

Lhomond, Brigitte, 1997. «Attirance et pratiques homosexuelles », in Hugues Lagrange et Brigitte Lhomond (éds), L'entrée dans la sexualité : le comportement des jeunes dans le contexte du sida. Paris : La Découverte, p. $183-226$.

McIntosh, Mary, 1968. « The homosexual role », Social Problems, 16 (2), p. 182-192.

MeAD, Margaret, 1950. " Some anthropological considerations concerning guilt », in Martin L. Reymert (éd.), Feelings and emotions. New York : McGraw-Hill, p. 362-373.

Messiah, Antoine et Emmanuelle Mouret-Fourme, 1993. «Homosexualité, bisexualité : éléments de sociobiographie sexuelle », Population, 48 (5), p. 1353-1380.

Moodie, T. Dunbar, Vivienne Ndatshe, et Sibuyi British, 1988. « Migrancy and male sexuality on the South African gold mines », Journal of Southern African Studies, 14 (2), p. 228-256.

Murray, Stephen O., 1997. «Explaining away same-sex sexualities : when they obtrude on anthropologists' notice at all », Anthropology Today, 13 (3), p. 2-5.

Niang, Cheikh Ibrahima, Placide Tapsoba, Ellen Weiss, Moustapha Diagne, Youssoupha Niang, Amadou Mody Moreau, Dominique Gomis, Abdoulaye Sidbé Wade, Karim Seck et Chris Castle, 2003. "'It's raining stones' : stigma, violence and HIV vulnerability among men who have sex with men in Dakar, Senegal », Culture, Health and Sexuality, 5 (6), p. 499-512.

Sagarin, Edward, 1976. «Prison homosexuality and its effect on post-prison sexual behavior », Psychiatry, 39, p. 45-257.

SHEPHERD, Gill, 1987. «Rank, gender, and homosexuality : Mombasa as a key to understanding sexual options », in Pat Caplan (éd., The cultural construction of sexuality. London : Tavistock Publications, p. 240-270.

Stein, Arlene, 1989. « Three models of sexuality : drives, identities and practices », Sociological Theory, 7 (1), p. $1-13$.

Sylla, Aliou, Amadigué Togo, Alou Dembélé avec la collaboration de Christophe Broqua, 2007. Analyse de la situation du groupe des hommes ayant des rapports sexuels avec d'autres hommes. Bamako : rapport de recherche Arcad-sida/Onusida.

Teunis, Niels, 2001. « Same-sex sexuality in Africa : a case study from Senegal », AIDS and Behavior, 5 (2), p. $173-182$.

VAN Wormer, Katherine, 1984. « Becoming homosexual in prison : a socialization process », Criminal Justice Review, 9, p. 22-27. 